\title{
REVISITING THE RECTANGULAR CONSTANT IN BANACH SPACES
}

\author{
M. BARONTI $\mathbb{D}^{\otimes}$, E. CASINI ${ }^{(i)}$ and P. L. PAPINI ${ }^{\circledR}$ \\ (Received 30 January 2021; accepted 24 February 2021; first published online 26 April 2021)
}

\begin{abstract}
Let $X$ be a real Banach space. The rectangular constant $\mu(X)$ and some generalisations of it, $\mu_{p}(X)$ for $p \geq 1$, were introduced by Gastinel and Joly around half a century ago. In this paper we make precise some characterisations of inner product spaces by using $\mu_{p}(X)$, correcting some statements appearing in the literature, and extend to $\mu_{p}(X)$ some characterisations of uniformly nonsquare spaces, known only for $\mu(X)$. We also give a characterisation of two-dimensional spaces with hexagonal norms. Finally, we indicate some new upper estimates concerning $\mu\left(l_{p}\right)$ and $\mu_{p}\left(l_{p}\right)$.
\end{abstract}

2020 Mathematics subject classification: primary 46B20; secondary 46B45, 46B99, 46C15.

Keywords and phrases: hexagonal norm, orthogonal vectors, rectangular constant, uniform nonsquareness.

\section{Introduction}

Let $X$ be a real Banach space. Let us denote by $B(X)$ and $S(X)$ the unit ball and the unit sphere, respectively.The vector $x$ is (Birkhoff-James) orthogonalto $y$ (which we denote by $x \perp y$ ) if $\|x\| \leq\|x+\lambda y\|$ for every real $\lambda$. In [8] (see also [9]), the rectangular constant was introduced:

$$
\mu(X)=\sup \left\{\frac{1+\lambda}{\|x+\lambda y\|}: x, y \in S(X), x \perp y, \lambda \geq 0\right\} .
$$

In [8], Joly proved that $\sqrt{2} \leq \mu(X) \leq 3$ and, for $\operatorname{dim}(X) \geq 3$, that $\mu(X)=\sqrt{2}$ if and only if $X$ is a Hilbert space. In [4], the equivalence was extended to two-dimensional spaces. Moreover, in [1], the following result was proved: $\mu(X)=3$ if and only if the space $X$ is nonuniformly nonsquare. We recall that a space $X$ is nonuniformly nonsquare (non-UNS for short) if for every $\epsilon>0$ there exist $x, y \in S(X)$ such that $\|x \pm y\|>2-\epsilon$.

In [6], Gastinel and Joly extended the definition of the rectangular constant: for $p \geq 1, x, y \in S(X)$ and $x \perp y$,

$$
\mu_{p}(x, y)=\sup _{\lambda \geq 0}\left\{\frac{\left(1+\lambda^{p}\right)^{1 / p}}{\|x+\lambda y\|}\right\}
$$

(C) Australian Mathematical Publishing Association Inc. 2021. This is an Open Access article, distributed under the terms of the Creative Commons Attribution licence (http://creativecommons.org/ licenses/by/4.0/), which permits unrestricted re-use, distribution, and reproduction in any medium, provided the original work is properly cited. 
and

$$
\mu_{p}(X)=\sup \left\{\mu_{p}(x, y): x, y \in S(X), x \perp y\right\} .
$$

We note that $\mu_{1}(X)=\mu(X)$. The following properties are proved in [6].

(A) We have $2^{(2-p) / 2 p} \leq \mu_{p}(X) \leq 3$. We remark that $\mu_{p}(X)$ is never smaller than 1 and so the left-hand inequality is meaningful only for $1 \leq p<2$. In Theorem 2.1 we will prove better estimates.

(B) If $\operatorname{dim}(X) \geq 3$, then $X$ is a Hilbert space if and only if $\mu_{p}(X)=2^{(2-p) / 2 p}$. By the preceding remark this is true only for $1 \leq p \leq 2$. In Theorem 2.1 we will revise this result by proving that, for $p \geq 2, X$ is a Hilbert space if and only if $\mu_{p}(X)=1$.

In Section 3 we will extend the characterisation of nonuniformly nonsquare spaces in terms of the parameter $\mu_{p}(X)$. More precisely we will prove that a space $X$ is non-UNS if and only if $\mu_{p}(X)=\left(1+2^{p}\right)^{1 / p}$ for every $p \geq 1$.

In Section 4 we will give a characterisation of two-dimensional spaces with symmetric orthogonality by using the parameter $\mu_{p}(X)$ and, finally, in the last section we will improve some upper bounds obtained in [5] for the parameter $\mu\left(l_{p}\right)$.

\section{Revisiting the Hilbert space characterisation}

As we have already remarked, Proposition 7.2.4 in [6] is correct only for $1 \leq p \leq 2$. In the following theorem we give the correct result for $p>2$.

THEOREM 2.1. Let $X$ be a real Banach space and $p \geq 1$.

(i) We have $\max \left\{1,2^{1 / p-1} \mu(X)\right\} \leq \mu_{p}(X) \leq \min \left\{\mu(X),\left(1+2^{p}\right)^{1 / p}\right\}$.

(ii) If $p \geq 2$ and $\operatorname{dim}(X) \geq 3$, then $X$ is a Hilbert space if and only if $\mu_{p}(X)=1$.

PROOF. From the inequality $a^{p}+b^{p} \leq(a+b)^{p} \leq 2^{p-1}\left(a^{p}+b^{p}\right)$, where $a$ and $b$ are nonnegative scalars, it follows immediately that $2^{1 / p-1} \mu(X) \leq \mu_{p}(X) \leq \mu(X)$. The inequality $\mu_{p}(X) \geq 1$ is trivial. Finally, since $\|x+\lambda y\| \geq 1$ and $\|x+\lambda y\| \geq|\lambda-1|$,

$$
\frac{1+\lambda^{p}}{\|x+\lambda y\|^{p}} \leq \min \left(1+\lambda^{p}, \frac{1+\lambda^{p}}{|\lambda-1|^{p}}\right) \leq 1+2^{p} .
$$

This concludes the proof of the first statement.

Suppose now that $\mu_{p}(X)=1$. If $x, y \in S(X)$ and $x \perp y$, then $\left(1+\lambda^{p}\right) /\|x+\lambda y\|^{p} \leq 1$. This implies that $\lambda \leq\|x+\lambda y\|$ or equivalently $1 \leq\|x / \lambda+y\|$ for every $\lambda>0$. Replacing $x$ by $-x$, for every $\lambda$ we have $\|\lambda x+y\| \geq 1=\|y\|$, which means that $y \perp x$. So, orthogonality is symmetric and so by [7] the space $X$ is a Hilbert space. It is easy to prove that if $X$ is a Hilbert space, then $\mu_{p}(X)=1$.

Therefore, the correct characterisation of Hilbert spaces in terms of $\mu_{p}(X)$ is the following: if $\operatorname{dim}(X) \geq 3$, then $X$ is a Hilbert space if and only if $\mu_{p}(X)=$ $\max \left\{1,2^{(2-p) / 2 p}\right\}$. 


\section{Uniformly nonsquare spaces}

In this section we extend Theorem 4 in [1]. We recall that the property that $X$ is non-UNS can equivalently be defined in the following way: for every $\epsilon>0$, there exist $x, y \in S(X)$ such that $\|x \pm y\|<1+\epsilon$.

THEOREM 3.1. The following conditions are equivalent:

(a) $X$ is non-UNS;

(b) for every $p \geq 1, \mu_{p}(X)=\left(1+2^{p}\right)^{1 / p}$;

(c) there exists $p \geq 1$ such that $\mu_{p}(X)=\left(1+2^{p}\right)^{1 / p}$.

Proof. ( $\Rightarrow$ a) If $\mu_{p}(X)=\left(1+2^{p}\right)^{1 / p}$, then, for every $\epsilon>0$, there exist $\lambda_{\epsilon}>0$ and $x_{\epsilon}, y_{\epsilon} \in S(X)$ with $x_{\epsilon} \perp y_{\epsilon}$ such that

$$
1+2^{p}-\epsilon \leq \frac{1+\lambda_{\epsilon}^{p}}{\left\|x_{\epsilon}+\lambda_{\epsilon} y_{\epsilon}\right\|^{p}} \leq 1+2^{p} .
$$

It is easy to show that this implies that $2^{p}-\epsilon<\lambda_{\epsilon}^{p}<2^{p}+\delta(\epsilon)$ with $\delta(\epsilon) \rightarrow 0$ when $\epsilon \rightarrow 0$. From this,

$$
\left\|x_{\epsilon}+\lambda_{\epsilon} y_{\epsilon}\right\| \leq\left(\frac{1+\lambda_{\epsilon}^{p}}{1+2^{p}-\epsilon}\right)^{1 / p} \leq\left(\frac{1+2^{p}+\delta(\epsilon)}{1+2^{p}-\epsilon}\right)^{1 / p}
$$

and so $1 \leq\left\|x_{\epsilon}+\lambda_{\epsilon} y_{\epsilon}\right\| \leq 1+\eta(\epsilon)$ with $\eta(\epsilon) \rightarrow 0$ when $\epsilon \rightarrow 0$. Next, $f(t)=\left\|x_{\epsilon}+t y_{\epsilon}\right\|$ is a convex function such that $1 \leq f(t), f(0)=1$ and $f\left(\lambda_{\epsilon}\right) \leq 1+\eta(\epsilon)$, so it follows that $1 \leq\left\|x_{\epsilon}+y_{\epsilon}\right\|<1+\eta(\epsilon)$. Let $z=\left(x_{\epsilon}+y_{\epsilon}\right) /\left\|x_{\epsilon}+y_{\epsilon}\right\|$. Then

$$
\begin{aligned}
\left\|z+y_{\epsilon}\right\|=\frac{\left\|x_{\epsilon}+y_{\epsilon}+\right\| x_{\epsilon}+y_{\epsilon}\left\|y_{\epsilon}\right\|}{\left\|x_{\epsilon}+y_{\epsilon}\right\|} & \leq\left\|x_{\epsilon}+\lambda_{\epsilon} y_{\epsilon}+\left(1+\left\|x_{\epsilon}+y_{\epsilon}\right\|-\lambda_{\epsilon}\right) y_{\epsilon}\right\| \\
& \leq\left\|x_{\epsilon}+\lambda_{\epsilon} y_{\epsilon}\right\|+\left|\left(1+\left\|x_{\epsilon}+y_{\epsilon}\right\|-\lambda_{\epsilon}\right)\right| \\
& \leq 1+\eta(\epsilon)+\left|1-\left\|x_{\epsilon}+y_{\epsilon}\right\|+\right| \lambda_{\epsilon}-2 \mid=1+\delta_{1}(\epsilon)
\end{aligned}
$$

with $\delta_{1}(\epsilon) \rightarrow 0$ when $\epsilon \rightarrow 0$. Similarly,

$$
\begin{aligned}
\left\|z-y_{\epsilon}\right\|=\frac{\left\|x_{\epsilon}+y_{\epsilon}-\right\| x_{\epsilon}+y_{\epsilon}\left\|y_{\epsilon}\right\|}{\left\|x_{\epsilon}+y_{\epsilon}\right\|} & \leq\left\|x_{\epsilon}+\left(1-\left\|x_{\epsilon}+y_{\epsilon}\right\|\right) y_{\epsilon}\right\| \\
& \leq\left\|x_{\epsilon}\right\|+\left|1-\left\|x_{\epsilon}+y_{\epsilon}\right\|\right| \leq 1+\eta(\epsilon) .
\end{aligned}
$$

So, $X$ is non-UNS.

$(\mathrm{a} \Rightarrow \mathrm{b})$ Let $X$ be non-UNS. Fix $\epsilon$ with $0<\epsilon<1 / 2$. There exist $x, y \in S(X)$ such that $\|x \pm y\|>2-\epsilon^{2}$. By convexity, $\|\lambda x \pm(1-\lambda) y\| \geq 1-2 \epsilon^{2}$ for every $\lambda \in[0,1]$. Moreover, $\|x+\epsilon y\|>1$. Indeed, $2-\epsilon^{2}<\|x+y\| \leq\|x+\epsilon y\|+1-\epsilon$ and so $\|x+\epsilon y\|>$ $1+\epsilon-\epsilon^{2}$. Let $F(\lambda)=\|\epsilon y+\lambda(x-(x+\epsilon y) /\|x+\epsilon y\|)\|$. It is easy to show that $F$ is a convex function with $F(1)=F(\|x+\epsilon y\|)$ and so there exists $\lambda_{0}>1$ such that $F$ attains its minimum. It follows that the two vectors $a=\epsilon y+\lambda_{0}(x-(x+\epsilon y) /\|x+\epsilon y\|)$ and 
$b=x-(x+\epsilon y) /\|x+\epsilon y\|$ are orthogonal. Moreover,

$$
\begin{aligned}
\|b\| & =\left(\frac{\|x+\epsilon y\|-1+\epsilon}{\|x+\epsilon y\|}\right)\left\|\frac{\|x+\epsilon y\|-1}{\|x+\epsilon y\|-1+\epsilon} x+\frac{\epsilon}{\|x+\epsilon y\|-1+\epsilon}(-y)\right\| \\
& \geq\left(\frac{\|x+\epsilon y\|-1+\epsilon}{\|x+\epsilon y\|}\right)\left(1-2 \epsilon^{2}\right) \geq\left(\frac{(1+\epsilon)\left\|\frac{x}{1+\epsilon}+\frac{\epsilon y}{1+\epsilon}\right\|-1+\epsilon}{1+\epsilon}\right)\left(1-2 \epsilon^{2}\right) \\
& \geq \frac{1-2 \epsilon^{2}}{1+\epsilon}\left((1+\epsilon)\left(1-2 \epsilon^{2}\right)-1+\epsilon\right)=\frac{1-2 \epsilon^{2}}{1+\epsilon} \epsilon\left(2-2 \epsilon-2 \epsilon^{2}\right)=\epsilon(2-\eta(\epsilon))
\end{aligned}
$$

with $\eta(\epsilon) \rightarrow 0$ as $\epsilon \rightarrow 0$. Finally, recalling that $\lambda_{0}>1$,

$$
\begin{aligned}
\mu_{p}^{p}(X) & \geq \frac{\|a\|^{p}+\lambda_{0}^{p}\|b\|^{p}}{\left\|a-\lambda_{0} b\right\|} \geq \frac{1}{\epsilon^{p}}\left(\left\|\epsilon y+\lambda_{0}\left(x-\frac{x+\epsilon y}{\|x+\epsilon y\|}\right)\right\|^{p}+\epsilon^{p}(2-\eta(\epsilon))^{p}\right) \\
& \left.\geq \frac{1}{\epsilon^{p}}\left(\lambda_{0}\left\|x-\frac{x+\epsilon y}{\|x+\epsilon y\|}\right\|-\epsilon\right)^{p}+\epsilon^{p}(2-\eta(\epsilon))^{p}\right) \\
& \geq \frac{1}{\epsilon^{p}}\left((\epsilon(2-\eta(\epsilon))-\epsilon)^{p}+\epsilon^{p}(2-\eta(\epsilon))^{p}\right)=(1-\eta(\epsilon))^{p}+(2-\eta(\epsilon))^{p} .
\end{aligned}
$$

We remark that it is easy to show (see [6]) that if $\mu(X)=3$ is attained, that is, if there exist $x$ and $y$ such that $x \perp y$ and $\mu_{1}(x, y)=3$, then there is a segment of length 2 on the unit sphere. (See also [10] for an extension of this result.) The space $X=\left(\prod_{n=2}^{\infty} l_{n}^{2}\right)_{2}$ is a non-UNS space but it is strictly convex (see [2, page 185]), so in this space $\mu(X)=3$ but it is not attained. This gives an affirmative answer to Remark 2.2 in [10].

\section{Symmetric orthogonality}

We have already remarked that if $\operatorname{dim}(X) \geq 3$, the symmetry of Birkhoff-James orthogonality implies that $X$ is a Hilbert space. However, there are two-dimensional spaces which are not Hilbert spaces but orthogonality is still symmetric. A simple example is the space $X$ with the 'hexagonal' norm, that is, the norm generated by a regular hexagon. An easy evaluation shows that $\mu_{p}(X)=2^{1 / p}$ for any $p \geq 1$. In this section we give a necessary and sufficient condition for a two-dimensional space $X$ to be isometric to a space with 'hexagonal' norm. We denote by $J_{x}^{+}(y)$ the right derivative of the norm at $x$, that is, $J_{x}^{+}(y)=\lim _{\lambda \rightarrow 0^{+}}(\|x+\lambda y\|-\|x\|) / \lambda$ and similarly by $J_{x}^{-}(y)$ for the left derivative. The following properties of $J_{x}^{ \pm}(y)$ are easy to prove.

LEMMA 4.1. For $x, y \in X$ :

(1) $J_{x}^{+}(y)=\sup \left\{f(y): f \in S\left(X^{*}\right), f(x)=\|x\|\right\}$;

(2) $J_{x}^{-}(y)=\inf \left\{f(y): f \in S\left(X^{*}\right), f(x)=\|x\|\right\}$;

(3) $J_{x}^{+}(x+y)=J_{x}^{+}(x)+J_{x}^{+}(y)$ and $J_{x}^{-}(x+y)=J_{x}^{-}(x)+J_{x}^{-}(y)$;

(4) $J_{x}^{+}(-y)=-J_{x}^{+}(y)$ and $J_{x}^{-}(-y)=-J_{x}^{-}(y)$;

(5) $J_{x}^{-}(y) \leq 0 \leq J_{x}^{+}(y)$ if and only if $x \perp y$. 
LEMMA 4.2. Let $x \perp y$ with $x, y \in S(X)$ and $p>1$. Let $\lambda_{0}$ be such that

$$
\mu_{p}^{p}(x, y)=\frac{1+\lambda_{0}^{p}}{\left\|x+\lambda_{0} y\right\|^{p}} .
$$

Then $x+\lambda_{0} y \perp \lambda_{0}^{p-1} x-y$.

Proof. Let $F(\lambda)=\left(1+\lambda^{p}\right) /\|x+\lambda y\|^{p}$ and suppose that $F\left(\lambda_{0}\right) \geq F(\lambda)$ for every $\lambda \geq 0$. Then

$$
F_{+}^{\prime}(\lambda)=\frac{p \lambda^{p-1}\|x+\lambda y\|^{p}-p\left(1+\lambda^{p}\right)\|x+\lambda y\|^{p-1} J_{x+\lambda y}^{+}(y)}{\|x+\lambda y\|^{2 p}} .
$$

So, $F_{+}^{\prime}\left(\lambda_{0}\right) \leq 0$ and, by Lemma 4.1 ,

$$
\left[\lambda_{0}^{p-1}\left\|x+\lambda_{0} y\right\|-\left(1+\lambda_{0}^{p}\right) J_{x+\lambda_{0} y}^{+}(y)\right]=\lambda_{0}^{p-1} J_{x+\lambda_{0} y}^{-}\left(x+\lambda_{0} y\right)+J_{x+\lambda_{0} y}^{-}\left(\left(-1-\lambda_{o}^{p}\right) y\right) \leq 0 .
$$

Moreover,

$$
J_{x+\lambda_{0} y}^{-}\left(\lambda_{0}^{p-1} x+\lambda_{0}^{p} y-y-\lambda_{0}^{p} y\right)=J_{x+\lambda_{0} y}^{-}\left(\lambda_{0}^{p-1} x-y\right) \leq 0 .
$$

Finally, in the same way, $J_{x+\lambda_{0} y}^{+}\left(\lambda_{0}^{p-1} x-y\right) \geq 0$ and this implies that $x+\lambda_{0} y \perp$ $\lambda_{0}^{p-1} x-y$.

THEOREM 4.3. Let $\operatorname{dim}(X)=2$ and suppose that Birkhoff-James orthogonality is symmetric. Then the following statements are equivalent:

(a) X has a 'hexagonal' norm;

(b) $\mu_{p}(X)=2^{1 / p}$ for every $p \geq 1$;

(c) there exists $p \geq 1$ such that $\mu_{p}(X)=2^{1 / p}$.

ProOF. Suppose that $\mu_{p}(X)=2^{1 / p}=\left(1+\lambda_{0}^{p}\right)^{1 / p} /\left\|x+\lambda_{0} y\right\|$. Since the orthogonality is symmetric,

$$
2=\frac{1+\lambda_{0}^{p}}{\left\|x+\lambda_{0} y\right\|^{p}} \leq 1+\lambda_{0}^{p}
$$

and

$$
2=\frac{1+\lambda_{0}^{p}}{\left\|x+\lambda_{0} y\right\|^{p}} \leq \frac{1+\lambda_{0}^{p}}{\lambda_{0}^{p}}
$$

and this implies that $\lambda_{0}=1$. So, $\|x+\lambda y\|=1$ for $\lambda \in[0,1]$ and, by Lemma 4.2, we obtain $x+y \perp x-y$. Consider the linear map $T: X \rightarrow \mathbb{R}^{2}$ with $T(x)=(1,0)$ and $T(y)=(-1,1)$ and define $\|T(z)\|\|=\| z \|$. Then $\|x+\lambda y\|=1$ implies that $\|(1-\lambda, \lambda)\|=1$ for $\lambda \in[0,1]$. Again, $\|\mid \lambda(-1,1)+(1-\lambda)(0,1)\|\|=\| y+2(1-\lambda) x \| \geq$ $\|y\|=1$ for $\lambda \in[0,1]$ and by convexity $\|\lambda(-1,1)+(1-\lambda)(0,1)\| \|=1$. Since $\|x+y\|=1=\|y\| \leq\|x+y+\lambda x\|$, it follows that $x \perp x+y$. Finally, we observe that $\|\mid \lambda(-1,1)+(1-\lambda)(-1,0)\|\|=\| x-\lambda(x+y) \| \geq 1$ and again, by convexity, we have $\||\lambda(-1,1)+(1-\lambda)(-1,0)|\|=1$ for every $\lambda \in[0,1]$. 
We conclude this section by showing that in the class of two-dimensional spaces with symmetric orthogonality we always have $\mu_{p}(H) \leq \mu_{p}(X) \leq 2^{1 / p}$, where $H$ denotes the Euclidean plane. As shown by Theorem 4.3, the upper bound is attained by the hexagonal norm.

THEOREM 4.4. Let $X$ be a two-dimensional space with symmetric orthogonality. Then $\mu_{p}(X) \leq 2^{1 / p}$.

PRoOF. Let $x, y \in S(X)$ and $x \perp y$ (and so $y \perp x$ ). Then

$$
\sup _{\lambda \geq 0} \frac{\left(1+\lambda^{p}\right)^{1 / p}}{\|x+\lambda y\|}=\sup _{\lambda>0} \frac{\left(1+\left(1 / \lambda^{p}\right)\right)^{1 / p}}{\|x+(1 / \lambda) y\|}=\sup _{\lambda \geq 0} \frac{\left(1+\lambda^{p}\right)^{1 / p}}{\|y+\lambda x\|},
$$

so that $\mu_{p}(x, y)=\mu_{p}(y, x)$. Let $\lambda_{0}$ be such that $\mu_{p}(x, y)=\left(1+\lambda_{0}^{p}\right)^{1 / p} /\left\|x+\lambda_{0} y\right\|$. Then

$$
\mu_{p}(x, y)=\frac{\left(1+\lambda_{0}^{p}\right)^{1 / p}}{\left\|x+\lambda_{0} y\right\|}=\frac{\left(1+\left(1 / \lambda_{0}\right)^{p}\right)^{1 / p}}{\left\|y+\left(1 / \lambda_{0}\right) x\right\|} \leq \mu_{p}(y, x)=\mu_{p}(x, y) .
$$

Since $\lambda_{0}$ or $1 / \lambda_{0}$ is less than or equal to 1 , this proves the theorem.

\section{Estimates in $l_{p}$ spaces}

The exact value of the parameter $\mu_{p}(X)$ is in general unknown. However, as we have already claimed, if $X$ is a Hilbert space, then $\mu(X)=\sqrt{2}$. It is also easy to obtain $\mu\left(l_{1}\right)=\mu\left(l_{\infty}\right)=3$. These results also follow from Theorem 3.1. Some bounds for $l_{p}$ spaces are given in [5]: $\mu\left(l_{p}\right) \leq(5+\sqrt{p}) /(1+\sqrt{p})$ for $1 \leq p \leq 2$ and $\mu\left(l_{p}\right) \leq 3-2 / 3 p$ for $p \geq 2$. In the next theorems, we will improve these estimates.

LEMMA 5.1. Let $p \geq 2, x, y \in S\left(l_{p}\right)$ and $x \perp y$. Then, for every $\lambda \geq 0$,

$$
\|x+\lambda y\|^{p} \geq 1+\frac{\lambda^{p}}{2^{p-1}-1} .
$$

PROOF. The proof follows easily if we prove that for every $N$,

$$
\|x+\lambda y\|^{p} \geq 1+\left(\sum_{n=1}^{N} 2^{n(1-p)}\right) \lambda^{p} .
$$

From the well-known Clarkson inequality,

$$
2\left(\|u\|^{p}+\|v\|^{p}\right) \leq\|u+v\|^{p}+\|u-v\|^{p},
$$

choosing $u=x+\lambda y$ and $v=\lambda y$,

$$
2\left(\|x+\lambda y\|^{p}+\lambda^{p}\right) \leq\|x+2 \lambda y\|^{p}+1 .
$$

Since $\|x+\lambda y\| \geq 1$, it follows that $\|x+2 \lambda y\|^{p} \geq 1+2 \lambda^{p}$. From this,

$$
\|x+\lambda y\|^{p} \geq 1+2^{1-p} \lambda^{p} .
$$


This shows that (5.1) is true for $N=1$. Let us suppose that (5.1) is true. Then, by (5.2),

$$
\begin{aligned}
\|x+2 \lambda y\|^{p} & \geq 2\|x+\lambda y\|^{p}+2 \lambda^{p}-1 \\
& \geq 2\left(1+\left(\sum_{n=1}^{N} 2^{n(1-p)}\right) \lambda^{p}\right)+2 \lambda^{p}-1=1+\left(\sum_{n=0}^{N} 2^{n(1-p)+1}\right) \lambda^{p} .
\end{aligned}
$$

This implies that

$$
\|x+\lambda y\|^{p} \geq 1+\left(\sum_{n=0}^{N} 2^{n(1-p)+1}\right) \frac{\lambda^{p}}{2^{p}}=1+\left(\sum_{n=1}^{N+1} 2^{n(1-p)}\right) \lambda^{p} .
$$

THEOREM 5.2. For $p \geq 2$,

$$
\mu\left(l_{p}\right) \leq\left(1+\left(2^{p-1}-1\right)^{1 /(p-1)}\right)^{(p-1) / p} .
$$

PROOF. Suppose that $x, y \in S\left(l_{p}\right)$ with $x \perp y$. Then, by Lemma 5.1,

$$
\mu(x, y)=\sup _{\lambda \geq 0} \frac{1+\lambda}{\|x+\lambda y\|} \leq \sup _{\lambda \geq 0} \frac{1+\lambda}{\left(1+\frac{\lambda^{p}}{2^{p-1}-1}\right)^{1 / p}} .
$$

It is easy to show that the function

$$
\phi(\lambda)=\frac{1+\lambda}{\left(1+\frac{\lambda^{p}}{2^{p-1}-1}\right)^{1 / p}}
$$

attains its maximum value for $\lambda=\left(2^{p-1}-1\right)^{1 /(p-1)}$, so

$$
\mu(x, y) \leq \phi\left(\left(2^{p-1}-1\right)^{1 /(p-1)}\right)=\left(1+\left(2^{p-1}-1\right)^{1 /(p-1)}\right)^{(p-1) / p} .
$$

LemMA 5.3. Let $1<p \leq 2, x, y \in S\left(l_{p}\right)$ and $x \perp y$. Then, for every $\lambda \geq 0$,

$$
\|x+\lambda y\|^{q} \geq 1+\frac{\lambda^{q}}{2^{q-1}-1},
$$

where $q$ and $p$ are conjugate indices.

PROOF. Starting from the inequality

$$
2^{q-1}\left(\|u\|^{q}+\|v\|^{q}\right) \geq\|u+v\|^{q}+\|u-v\|^{q},
$$

the proof follows with similar arguments to those in Lemma 5.1.

THEOREM 5.4. For $1<p \leq 2$,

$$
\mu\left(l_{p}\right) \leq\left(1+\left(2^{1 /(p-1)}-1\right)^{p-1}\right)^{1 / p} .
$$

PROOF. The proof of the theorem is similar to that of Theorem 5.2 with the help of Lemma 5.3. 
THEOREM 5.5. For $1<p \leq 2$,

$$
\mu\left(l_{p}\right) \leq \sqrt{\frac{p}{p-1}} .
$$

Proof. For $f, g \in l_{p}$, we use the inequality (see [3])

$$
2\|f\|^{2}+2\|g\|^{2} \geq\|f+g\|^{2}+(p-1)\|f-g\|^{2} .
$$

Let $x, y \in S\left(l_{p}\right)$ with $x \perp y$. If $f=\frac{1}{2} x+\lambda y$ and $g=\frac{1}{2} x$,

$$
2\left\|\frac{x}{2}+\lambda y\right\|^{2}+2\left\|\frac{x}{2}\right\|^{2} \geq\|x+\lambda y\|^{2}+(p-1)\|\lambda y\|^{2}
$$

or equivalently

$$
\|x+2 \lambda y\|^{2} \geq 2\|x+\lambda y\|^{2}+2(p-1) \lambda^{2}-1 .
$$

Since $\|x+\lambda y\| \geq 1$, we obtain $\|x+2 \lambda y\|^{2} \geq 1+2(p-1) \lambda^{2}$ and, from this,

$$
\|x+\lambda y\|^{2} \geq 1+\frac{p-1}{2} \lambda^{2} .
$$

It is now easy to prove by induction that

$$
\|x+\lambda y\|^{2} \geq 1+\frac{2^{n}-1}{2^{n}}(p-1) \lambda^{2}
$$

and so $\|x+\lambda y\|^{2} \geq 1+(p-1) \lambda^{2}$. This last inequality yields

$$
\mu(x, y)=\sup _{\lambda \geq 0} \frac{1+\lambda}{\|x+\lambda y\|} \leq \sup _{\lambda \geq 0} \frac{1+\lambda}{\left(1+(p-1) \lambda^{2}\right)^{1 / 2}} .
$$

Finally, simple calculations show that, for $\lambda>0$,

$$
\frac{1+\lambda}{\left(1+(p-1) \lambda^{2}\right)^{1 / 2}} \leq \sqrt{\frac{p}{p-1}} .
$$

From the last two theorems,

$$
\mu\left(l_{p}\right) \leq \min \left(\left(1+\left(2^{1 /(p-1)}-1\right)^{p-1}\right)^{1 / p}, \sqrt{\frac{p}{p-1}}\right) .
$$

A numerical evaluation shows that

$$
\left(1+\left(2^{1 /(p-1)}-1\right)^{p-1}\right)^{1 / p} \leq \sqrt{\frac{p}{p-1}}
$$

for $1<p \leq p_{0}$ with $p_{0} \simeq 1.188$.

With the aid of Lemmas 5.1 and 5.3, we also obtain some estimates for $\mu_{p}\left(l_{p}\right)$.

THEOREM 5.6. For $p \geq 2$,

$$
\mu_{p}\left(l_{p}\right) \leq\left(2^{p-1}-1\right)^{1 / p}
$$


ProOF. Suppose that $x, y \in S\left(l_{p}\right)$ with $x \perp y$. Then, by Lemma 5.1,

$$
\mu_{p}^{p}(x, y)=\sup _{\lambda \geq 0} \frac{1+\lambda^{p}}{\|x+\lambda y\|^{p}} \leq \sup _{\lambda \geq 0} \frac{1+\lambda^{p}}{1+\frac{\lambda^{p}}{2^{p-1}-1}}=\sup _{\lambda \geq 0} \phi(\lambda) \quad \text { (say). }
$$

It is easy to show that the function $\phi(\lambda)$ is increasing, so

$$
\mu_{p}(x, y) \leq \lim _{\lambda \rightarrow \infty}\left(\frac{1+\lambda^{p}}{1+\frac{\lambda^{p}}{2^{p-1}-1}}\right)^{1 / p}=\left(2^{p-1}-1\right)^{1 / p} .
$$

THEOREM 5.7. For $1<p<2$,

$$
\mu_{p}\left(l_{p}\right) \leq\left(1+\left(2^{1 /(p-1)}-1\right)^{(p-1) /(2-p)}\right)^{(2-p) / p} .
$$

Proof. The proof is similar to that of Theorem 5.6. Suppose that $x, y \in S\left(l_{p}\right)$ with $x \perp y$. Then, by Lemma 5.3,

$$
\|x+\lambda y\|^{q} \geq 1+\frac{\lambda^{q}}{2^{q-1}-1},
$$

where $q$ and $p$ are conjugate indices. Consequently,

$$
\mu_{p}^{p}(x, y)=\sup _{\lambda \geq 0} \frac{1+\lambda^{p}}{\|x+\lambda y\|^{p}} \leq \sup _{\lambda \geq 0} \frac{1+\lambda^{p}}{\left(1+\frac{\lambda^{q}}{2^{q-1}-1}\right)^{p / q}}=\sup _{\lambda \geq 0} \phi(\lambda) \quad \text { (say). }
$$

Finally, the maximum of the function $\phi(\lambda)$ is the upper bound in the theorem.

In [6], Gastinel and Joly proved that $\mu\left(l_{p}\right)=\mu\left(l_{p}^{2}\right)$, where $l_{p}^{2}$ is the two-dimensional $l_{p}$ space and they gave a table with some numerical estimates of the rectangular constant for $l_{p}^{2}$ spaces. In their subsequent Remark 11.4.1, they suggested that probably $\mu\left(l_{p}\right)=\mu\left(l_{q}\right)$, where $p$ and $q$ are conjugate. We conclude with a similar table (see Table 1) obtained with more accurate calculations, which shows instead that, in general, they are different.

TABLE 1. Values of $\mu\left(l_{p}\right)$ and $\mu\left(l_{q}\right)$.

\begin{tabular}{lccc}
\hline$p$ & $\mu\left(l_{p}\right)$ & $q$ & $\mu\left(l_{q}\right)$ \\
\hline 2 & 1.4142 & 2 & 1.4142 \\
3 & 1.7285 & $3 / 2$ & 1.6554 \\
4 & 1.9337 & $4 / 3$ & 1.8264 \\
5 & 2.0772 & $5 / 4$ & 1.9554 \\
10 & 2.4328 & $10 / 9$ & 2.3099 \\
15 & 2.5826 & $15 / 14$ & 2.4742 \\
30 & 2.7598 & $30 / 29$ & 2.6819 \\
50 & 2.8429 & $50 / 49$ & 2.7854 \\
100 & 2.9131 & $100 / 99$ & 2.8771 \\
\hline
\end{tabular}




\section{References}

[1] M. Baronti, 'On some parameters of normed spaces', Boll. Unione Mat. Ital. B (5) 18(3) (1981), 1065-1085.

[2] B. Beauzamy, Introduction to Banach Spaces and their Geometry, 2nd edn, North-Holland Mathematics Studies, 68 (North-Holland, Amsterdam, 1985).

[3] W. L. Bynum, 'Weak parallelogram laws for Banach spaces', Canad. Math. Bull. 19(3) (1976), 269-275.

[4] M. del Río and C. Benítez, 'The rectangular constant for two-dimensional spaces', J. Approx. Theory 19(1) (1977), 15-21.

[5] J. Desbiens, 'Constante rectangle et biais d'un espace de Banach', Bull. Aust. Math. Soc. 42(3) (1990), 465-482.

[6] N. Gastinel and J. L. Joly, 'Condition numbers and general projection method', Linear Algebra Appl. 3 (1970), 185-224.

[7] R. C. James, 'Orthogonality and linear functionals in normed linear spaces', Trans. Amer. Math. Soc. 61 (1947), 265-292.

[8] J. L. Joly, 'Caractérisations d'espaces hilbertiens au moyen de la constante rectangle', J. Approx. Theory 2 (1969), 301-311.

[9] J. L. Joly, 'La constante rectangle d'un espace vectoriel normé', C. R. Acad. Sci. Paris Sér. A-B 268 (1969), A36-A38.

[10] K. Paul, P. Ghosh and D. Sain, 'On rectangular constant in normed linear spaces', J. Convex Anal. 24(3) (2017), 917-925.

M. BARONTI, Dipartimento di Matematica, Università di Genova, Via Dodecaneso 35,

16100 Genova, Italy

e-mail: baronti@dima.unige.it

E. CASINI, Dipartimento di Scienza e Alta Tecnologia,

Università dell'Insubria, Via Valleggio 11, 22100 Como, Italy

e-mail: emanuele.casini@uninsubria.it

P. L. PAPINI, Via Martucci 19, 40136 Bologna, Italy

e-mail: pierluigi.papini@unibo.it 